\title{
DUCHENNE GENE CARRIER AS CAUSE OF ASYMPTOMATIC HYPERCKEMIA
}

\author{
Diogo Fraxino de Almeida ${ }^{2,3}$, Aluisio Cláudio Mentor Couto Melo Jr², \\ Paulo Rogério M. de Bittencourt ${ }^{7}$
}

Asymptomatic hyperCKemia can be a clinical challenge for physicians, although its long term prognosis seems benign'. The causes usually include heavy exercise practice, muscle injury, drug-induced myopathy, chronic alcoholism, hypothyroidism, clinically silent myopathies, and susceptibility to malignant hyperthermia. A frequently missed cause in women is the carrier status of the Duchenne muscular dystrophy ${ }^{2}$.

We report a case and discuss its investigational approach.

\section{CASE}

A 15 year-old girl was referred to us with asymptomatic hyperCKemia after an extensive prior investigation. The hyperCKemia was found after liver enzymes had been found mildly eleveted during a fever work-up. There was no history of exercise intolerance, muscle injury, neuromuscular disease in the family, myotoxic drug use or personal and family history of anesthetic complications. She has an asymptomatic brother as well as no other family member has any history of neurological disorders. Resting CPK levels varied from 500 to $1400 \mathrm{U} / \mathrm{L}$ in the last three years. An electrophysiological study showed mild chronic myopathy represented by normal nerve conduction studies, no abnormal spontaneous activity, and moderate amount of polyphasic small amplitude and short duration motor unit potentials (MUP) with early recruitment (Fig 1). An extensive laboratory work-up was normal including $C B C$, sedimentation rate, ANA, TSH, free T4, PTH and electrolytes. A muscle biopsy performed in other medical center showed non-specific myopathic changes and a diagnosis of myotonic myopathy had been proposed, despite the absence of clinical or electrophysiological myotonia and negative genetic testing. A deletion in the exons 46-47 of the dystrophin gene was detected by Southern-blotting and PCR techniques. The CPK levels in other family members were normal. A cardiologic evaluation including routine as well as 24-hours electrocardiogram and echocardiogram were normal. Further genetic study in family members was denied. Genetic counseling and clinical follow-up are being provided on a regular basis.

The patient parents have agreed with this publication.

\section{DISCUSSION}

Idiopathic hyperCKemia $(\mathrm{IH})$ is defined as elevated serum CK and no clinical, electrophysiological or pathological evidence of neuromuscular diseases ${ }^{3}$. Further exclusion criteria accepted nowadays include thyroid and parathyroid disease, pregnancy, muscle injury, heavy physical activity, family history of neuromuscular disease and use

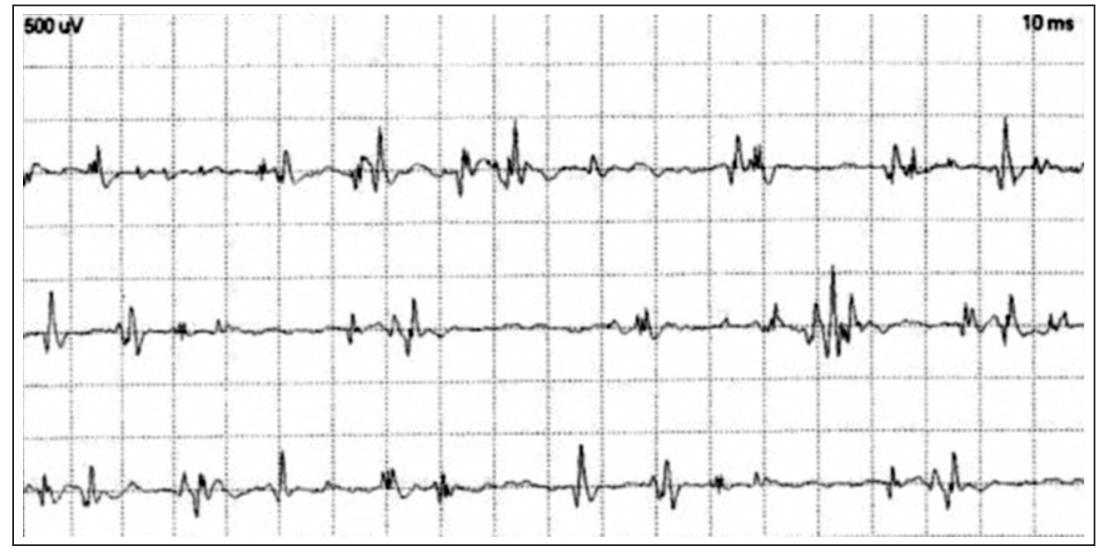

Fig 1. Small amplitude and short duration (SASD) motor unit potentials and polyphasic small amplitude motor unit potentials in deltoid muscle.

\section{GENE DA DISTROFIA DE DUCHENNE EM PORTADORA ASSINTOMÁTICA COMO CAUSA DE CPK ELEVADA}

'UNINEURO, Unidade de Neurologia Clínica, Curitiba PR, Brazil; ' ${ }^{2}$ MMG LAB, Nossa Senhora das Graças Hospital (HNSG), Curitiba PR, Brazil; ${ }^{3}$ University Hospital - Universidade Estadual de Maringá, Maringá PR, Brazil.

Received 18 December 2007, received in final form 25 March 2008. Accepted 31 March 2008.

Dr. Diogo Fraxino de Almeida - Avenida Independência 258/405 - 87015-020 Maringá PR - Brasil. E-mail: diogofraxino@hotmail.com 


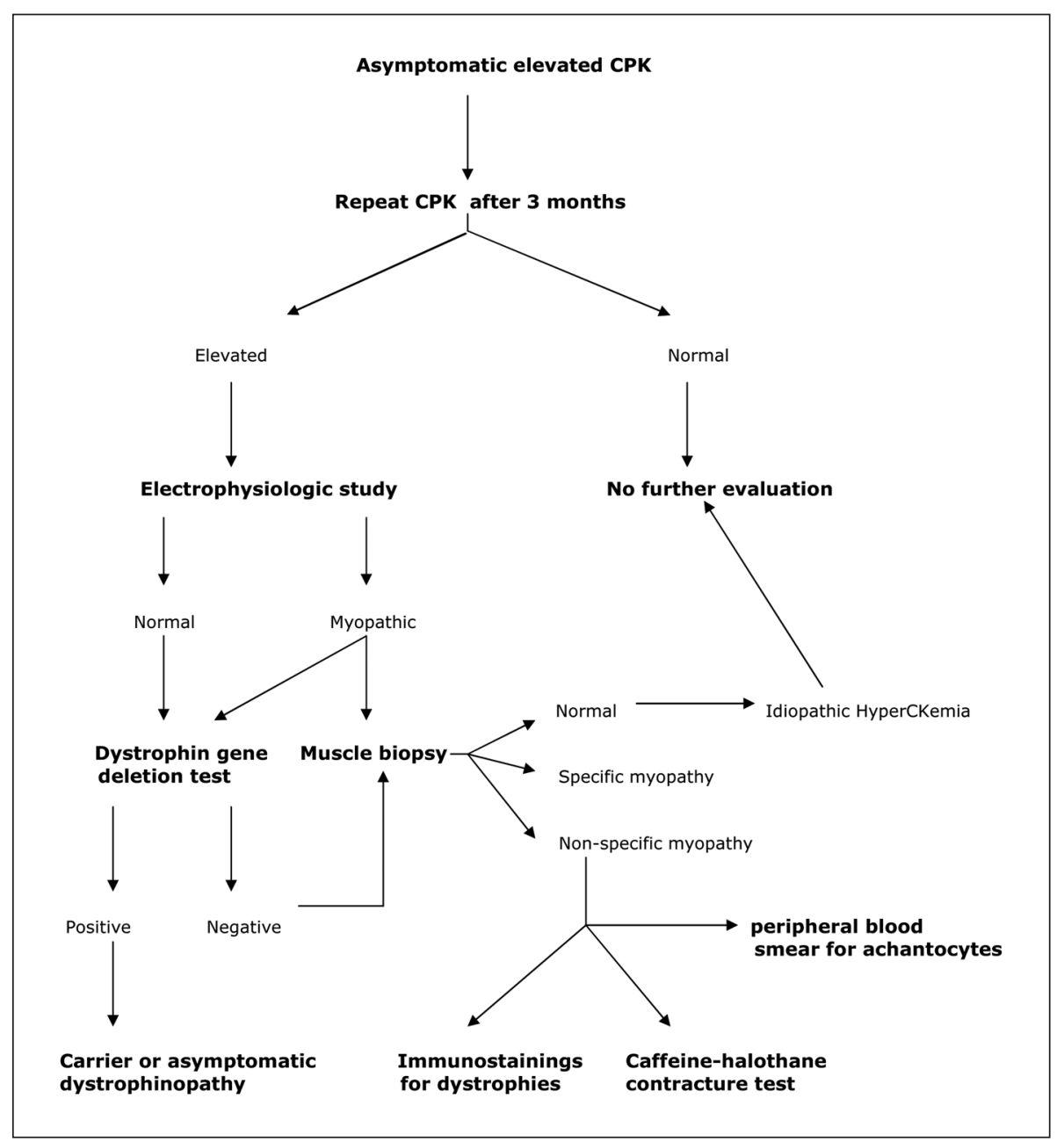

Fig 2. Suggested approach to the asymptomatic hyperCKemia.

of myotoxic medications ${ }^{4}$. Some authors believe that the diagnosis of $\mathrm{IH}$ only can be made after a normal muscle biopsy ${ }^{5}$. Our patient did not fulfill the criteria for $\mathrm{IH}$, since she had non-specific myopathic changes in the needle EMG and muscle biopsy. A better term to be used in this context could be "asymptomatic pathologic hyperCKemia". In fact, most patients with persistent asymptomatic elevated CPK levels have an underlying muscle disease ${ }^{7}$, usually with a benign course ${ }^{1}$, although in a minority of cases the abnormality can precede an ominous neuromuscular disease ${ }^{6}$. Our patient had received a diagnosis of myotonic dystrophy in other medical center based on the presence of excessive central nuclei in the muscle biopsy, despite the absence of clinical and electrophysiological myotonia and negative genetic testing for myotonic dystrophy.

Two usually underdiagnosed conditions associated with idiopathic or "asymptomatic pathologic" hyperCKemia are malignant hyperthermia susceptibility and dystrophin gene carrier status. Less frequently, caveolin-3 gene mutation and absence of dysferlin and sarcoglycans are found in patients with asymptomatic hyperCKemia ${ }^{6}$, as well as patients with neuroacanthocytosis syndromes, including autossomal-recessive chorea-acanthocytosis and $\mathrm{X}$-linked McLeod syndrome ${ }^{8}$. In all these diseases, the CK elevation can predate by many years the neurological symptoms.

Malignant hyperthermia (MH) is a rare autosomal dominant disease associated with halogenated anesthetics and depolarizing neuromuscular blocking agents use. A study found $\mathrm{MH}$ susceptibility in half of patients with possible idiopathic hyperCKemia and recommended the caffeine-halothane contracture testing in all asymptomatic patients who undergo muscle biopsy due to persistently elevated $\mathrm{CPK}^{9}$. Just a few centers in Brazil are able to perform this procedure.

Duchenne carrier status occurs in women with or without family history of Duchenne muscular dystrophy. Only a minority of them have "important weakness" and, when it is present, they are coined "manifesting genetic carriers". Elevated CPK levels occur in up to $70 \%$ of carrier women of dystrophin gene ${ }^{10}$. Dystrophin immunohystochemical staining should be included in the routine muscle biopsy 
study in patients with hyperCKemia to rule out Becker muscular dystrophy in men or dystrophin gene carrier status in women, regardless of family history ${ }^{5,11}$. Typical findings include partially dystrophin-deficient fibers and mosaic pattern (dystrophin positive and dystrophin negative fibers) of staining ${ }^{12}$. If such staining had been done in the muscle analysis of our patient, the correct diagnosis could have not been missed.

Genetic analysis of common deletions in the dystrophin gene is a feasible alternative to avoid muscle biopsy in patients with hyperCKemia. Molecular techniques detect carriers and permit prenatal diagnosis in $95 \%$ of the patients $^{13}$. The dystrophin gene is localized at the chromosome Xp21 and has 79 exons. About 70\% of the patients have intragenic deletions of one or more exons, usually at the proximal or central regions of the gene. The deletions can be detected by PCR or Southern blotting using the patient's blood. The diagnostic confirmation of the carrier status in females is hard by the presence of a normal copy of the gene in the other $\mathrm{X}$ chromosome and the relatively high incidence of point mutations. Another alternative is performing a DNA based linkage analysis using several polymorphic intragenic short tandem repeat markers. This method is a powerful helper in the diagnosis of the carrier status detection of deletional as well as non-deletional dystrophinopathy families. However, it requires a blood sample of the proband and other family members. In suspected patients with negative genetic tests, a muscle biopsy with dystrophin immunohistochemistry is indicated ${ }^{14}$.

In dystrophin gene carrier women, periodid cardiologic evaluation is indicated, since some patients can show abnormalities in heart conduction and function ${ }^{15}$. In the majority of the carriers cardiac abnormalities are asymptomatic, but close monitoring is recommended.
A suggested step by step approach for asymptomatic hyperCKemia can be seen in the Figure 2. When all such work up is negative, asymptomatic hyperCKemia is reasonable and the patient can be assured that the prognosis is benign.

\section{REFERENCES}

1. Reijneveld JC, Notermans NC, Linssen WHJP, Wokke JHJ. Benign prognosis in idiopathic hyperCKemia. Muscle \& Nerve 2000;23:575-579.

2. Hoffman EP, Arahata K, Minetti C, Bonilla E, Rowland LP. Dystrophinopathy in isolated cases of myopathy in females. Neurology 1992; 42:967-975.

3. Rowland LP, Willner J, DiMauro S, Miranda A. Approaches to the membrane theory of Duchenne muscular dystrophy. In Angelini C, Danieli GA, Fontanari D (Eds). Muscular dystrophy: advances and new trends. Amsterdam, Excerpta Medica, 1980:3-13.

4. Brewster LM, de Visser M. Persistent hyperCKemia: fourteen patients studied in retrospect. Acta Neurol Scand 1988;77:60-63.

5. Prelle A, Tancredi L, Sciacco M, et al. Retrospective study of a large population of patients with asymptomatic or minimally symptomatic raised serum creatine kinase levels. J Neurol 2002;249:305-311.

6. Dabby R, Sadeh M, Herman O, et al. Asymptomatic or minimally symptomatic hyperCKemia: histopathologic correlation. IMAJ 2006;8:110-113.

7. Joy JL, Oh SJ. Asymptomatic hyperCKemia: an electrophysiologic and histopathologic study. Muscle \& Nerve 1989;12:206-209.

8. Walker RH, Peters JJ, Jung HH, Danek A. Diagnostic evaluation of clinically normal subjects with chronic hyperCKemia. Neurology 2007;68: 535-536.

9. Weglinski MR, Wedel DJ, Engel AG. Malignant hyperthermia testing in patients with persistently increased serum creatine kinase (CK) levels. Anesth Analg 1997;84:1038-1041.

10. Tachi N, Wakai S, Yutoh Y, Chiba S, Miura J. Asymptomatic hyperCKemia: detection of an isolated carrier of Duchenne muscular dystrophy. J Child Neurol 1990;5:351-353.

11. Oliveira ASB, Gabbai AA, Schmidt B, et al. Carrier detection of Duchenne and Becker muscular dystrophy using muscular dystrophin immunohistochemistry. Arq Neuropsiquiatr 1992;50:478-485.

12. Bonilla E, Schmidt B, Samitt CE, et al. Normal and dystrophin-deficient muscle fibers in carriers of the gene for Duchenne muscular dystrophy. Am J Pathol 1988;133:440-445.

13. Panigrahi I, Mitall B. Carrier detection and prenatal diagnosis in Duchenne/Becker muscular dystrophy. Indian Pediatr 2001;38:631-639.

14. Werneck LC, Scola RH, Maegawa GH, Werneck MC. Comparative analysis of PCR-deletion detection and immunohistochemistry in Brazilian Duchenne and Becker muscular dystrophy patients. Am J Med Genet 2001;103:115-120.

15. Hoogerwaard EM, Bakker E, Ippel PF, et al. Signs and symptoms of Duchenne muscular dystrophy and Becker muscular dystrophy among carriers in The Netherlands: a cohort study. Lancet 1999;353:2116-2119. 